\title{
Pleiotropic Effects of a Butyrolactone-type Autoregulator on Mutants of Streptomyces griseus Blocked in Cytodifferentiation
}

\author{
By UDO GRÄFE, * GÜNTHER REINHARDT, DIETRICH KREBS, \\ INGE ERITT AND W. F. FLECK \\ Central Institute of Microbiology and Experimental Therapy, GDR Academy of Sciences, \\ PO Box 73, 6900 Jena, GDR
}

(Received 13 Iuly 1983; revised 10 October 1983)

Mutants of Streptomyces griseus blocked in cytodifferentiation regained their capacity to form
differentiated mycelia and/or anthracycline pigments in the presence of butyrolactone-
type autoregulatory effectors such as trans-2-(6'-methylheptanol-1'-yl)-3-hydroxymethyl-4-
butanolide. In the pertinent indicator strains, the effect has been correlated with the increase of
lipid synthesis, with changes in the composition of lipid fraction and with the restoration of the
production of neutral proteinases. The results suggest that autoregulatory butyrolactones from
streptomycetes stimulate cytodifferentiation of their producers at an early stage of development.

\section{INTRODUCTION}

Differentiation of streptomycetes, for instance the formation of aerial mycelia and/or antibiotics, has been shown to require the appearance of endogenous signal molecules called bioregulators or autoregulators (Khokhlov, 1982; Biro et al., 1980; Pogell, 1979). These factors enable cytodifferentiation of asporogenous, antibiotic-negative mutants which are blocked in the production of the autoregulatory effectors. Elucidation of the mode of action of the autoregulators in the cells of susceptible indicator strains could provide further information on the regulation of the cell cycle of these industrially important micro-organisms (Pogell, 1979). Recently, we reported on the inducing effect of A-factor (I) (Kleiner et al., 1977; Khokhlov, 1982) on the morphological development of surface cultures of Streptomyces griseus JA 5142/86, an asporogenous, leukaemomycin- (anthracycline-) negative, autoregulator-negative mutant (Amy- $\mathrm{Lkm}^{-} \mathrm{Are}^{-}$) of the parental sporulating, leukaemomycin-producing, autoregulatorpositive strain $S$. griseus JA 5142 (Amy $^{+} \mathrm{Lkm}^{+}$Are ${ }^{+}$; Eritt et al., 1982; Gräfe \& Eritt, 1983). Compound $\mathrm{I}$ has been shown to reconstitute both the formation of spores and anthracyclines (leukaemomycin) in surface cultures of the blocked mutant 86 when added to the culture agar at time zero (Gräfe et al., 1981). Inducers of cytodifferentiation of this mutant strain structurally

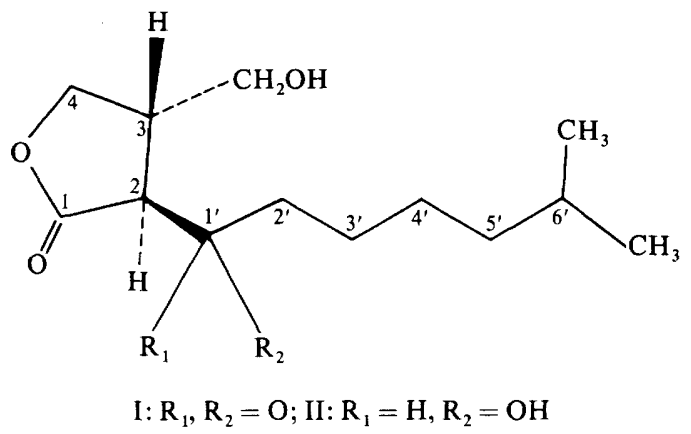


related to I have been generated by many other, even taxonomically different, streptomycetes (Eritt et al., 1983, 1984; Gräfe et al., 1982 b; 1983a). In the present work we studied the effect of one of these interspecific inducer molecules, trans-2-(6'-methylheptanol-1'-yl)-3-hydroxymethyl4-butanolide (II), on the development of submerged cultures of blocked mutants of anthracycline-producing strains of $S$. griseus incapable of producing the endogenous signal compound I. The aim was to correlate the induction of secondary metabolism and/or morphological changes with alterations in the rate of synthesis and the composition of mycelial lipids, because it has been anticipated that functional changes occur throughout the cell cycle of streptomycetes at the level of the cytoplasmic membrane (Chater \& Merrick, 1976; Kalakoutski \& Agre, 1976; Hopwood, 1983).

\section{METHODS}

Chemicals. NADP was supplied by Arzneimittelwerk (Dresden, GDR); azocasein, phenylmethylsulphonyl fluoride and standards of the fatty acid methyl esters were purchased from Serva (Heidelberg, FRG); phosphatidylethanolamine and cardiolipin were from Koch-Light; $\left[\mathrm{U}-{ }^{14} \mathrm{C}\right]$ acetate $\left(2 \times 10^{3} \mathrm{MBq} \mathrm{mmol}{ }^{-1}\right)$ and $\mathrm{KH}_{2}{ }^{32} \mathrm{PO}_{4}$ were from Isocommerz (Berlin, GDR); and X-ray film was from Orwo (GDR).

Organisms and cultivation conditions. The anthracycline-producing parental strains $S$. griseus JA 5142 (identical with ZIMET 43689) and JA 3933 were obtained from the strain collection of the Central Institute of Microbiology and Experimental Therapy, Jena, GDR. Both strains produced an almost identical mixture of anthracycline pigments (leukaemomycin), though they were isolated from different samples of soil (Fleck \& Strauss, 1975).

The blocked indicator mutants 86 (JA 5142/86) and 15 (JA 3933/15) were isolated after mutagenic treatment of spores of the ancestral strains JA 5142 and JA 3933, respectively, with $N$-nitroso- $N$-methylguanidine (NMG) and UV light by means of the screening procedure described previously (Eritt et al., 1982). All cultures were started from a stock of freeze-dried organisms and were propagated and maintained on AL53 medium (Eritt et al., 1982) containing $\left(1^{-1}\right): 3 \mathrm{~g}$ sucrose; $15 \mathrm{~g}$ dextrin; $0.1 \mathrm{~g}$ urea; $1 \mathrm{~g}$ yeast extract; $5 \mathrm{~g}$ bacto-peptone (Difco); $0.5 \mathrm{~g} \mathrm{NaCl}$; $0.5 \mathrm{~g} \mathrm{KH}_{2} \mathrm{PO}_{4} ; 0.01 \mathrm{~g} \mathrm{FeSO}_{4} .7 \mathrm{H}_{2} \mathrm{O} ; 15 \mathrm{~g}$ agar. The $\mathrm{pH}$ was 7.8 (after sterilization). Inocula were prepared by seeding lawns of $10 \mathrm{~d}$ surface mycelia into Hickey-Tresner's medium containing $\left(1^{-1}\right): 10 \mathrm{~g}$ dextrin $; 1 \mathrm{~g}$ yeast extract; $1 \mathrm{~g}$ beef extract (Difco); $2 \mathrm{~g}$ casein digest, $\mathrm{N}-\mathrm{Z}$ amine A (Difco); $0.2 \mathrm{~g} \mathrm{CoCl}_{2} .7 \mathrm{H}_{2} \mathrm{O}$. The pH was 6.5 (after sterilization). Inocula ( $48 \mathrm{~h}$ ) were seeded in $80 \mathrm{ml}$ of the same medium in $500 \mathrm{ml}$ flasks. All cultivations were carried out on rotary shakers $\left(240\right.$ r.p.m., $\left.29^{\circ} \mathrm{C}\right)$.

The autoregulatory effector II was substituted completely for the natural autoregulator (I) of the parental strains JA 5142 and JA 3933 because it has not been possible to isolate I from these ancestral strains in the required amount and purity for these investigations (Gräfe \& Eritt, 1983). According to NMR data, samples of II isolated from Streptomyces viridochromogenes (Gräfe et al., 1982b) were $>90 \%$ pure. At zero time, an aqueous, sterile solution of II was added to both inoculum and main cultures to give a final concentration of $10 \mu \mathrm{g} \mathrm{ml}^{-1}$.

Analytical procedures. Mycelial dry weight was estimated gravimetrically. Protein concentration in mycelial extracts was measured by the method of Lowry. Disintegration of mycelia was carried out in $0.1 \mathrm{M}$-Tris buffer $\mathrm{pH} 7.4$ by three $30 \mathrm{~s}$ sonic treatments $\left(0^{\circ} \mathrm{C}\right)$ with a Labsonic 1510 instrument (Braun, FRG). For the enzyme assays the cell debris was removed by centrifuging at $22000 \mathrm{~g}$ for $15 \mathrm{~min}$ and the supernatant used. For the measurement of the cytochrome spectra, centrifugation was carried out for $5 \mathrm{~min}$ at $2000 \mathrm{~g}$. Redox difference spectra of cytochromes $\left(\mathrm{Na}_{2} \mathrm{~S}_{2} \mathrm{O}_{3}\right.$ versus $\mathrm{H}_{2} \mathrm{O}_{2}$ treated samples) were recorded by means of a Cary 117 instrument. Lipids were extracted from freshly harvested mycelia with $\mathrm{CHCl}_{3} /$ methanol $(2: 1, \mathrm{v} / \mathrm{v})$, dried and weighed as described previously (Gräfe et al., 1982c). The methyl esters of fatty acids were obtained by the treatment of lipids with $2.5 \% \mathrm{HCl}$ in methanol. TLC of lipids was carried out on silica gel sheets (precoated, Merck) using either $\mathrm{CHCl}_{3} /$ methanol $/ \mathrm{H}_{2} \mathrm{O}\left(65: 25: 4\right.$, by vol.) or in three subsequent runs of the same plate, $\mathrm{CHCl}_{3} /$ methanol $/ \mathrm{H}_{2} \mathrm{O}$ $\left(65: 25: 4\right.$, by vol.), then $\mathrm{CHCl}_{3} / n$-hexane $(3: 1, \mathrm{v} / \mathrm{v})$ and then $\mathrm{CCl}_{4}$. Authentic standards of phospholipids were run in parallel. The presence of ornithinolipid was established through the hydrolysis of lipids as described previously (Gräfe et al., 1982c). Gas chromatography of fatty acid methyl esters was done with a gas chromatograph model GCHF 18:3 (VEB Chromatron, Berlin, GDR) equipped with a flame ionization detector. Glass columns $(3 \mathrm{~m} \times 3 \mathrm{~mm}$ i.d.) were used, filled with $3 \%(\mathrm{w} / \mathrm{w})$ diethyleneglycol-succinate polyester (DEGS, Serva) on Chromosorb G (80-100 mesh, Serva) and operated isothermically at $165^{\circ} \mathrm{C}$. The injector temperature was $200{ }^{\circ} \mathrm{C}$, and nitrogen was the carrier gas $\left(35 \mathrm{ml} \mathrm{min}{ }^{-1}\right)$. Peaks were identified through their retention times as compared with those of authentic standards.

Enzyme assays. NAD(P)-glycohydrolase (EC 3.2.2.5) was assayed according to Voronina et al. (1978) as described previously (Gräfe et al., 1981). Neutral proteinase and serine proteinase (EC 3.4 17.1) were measured according to Ginther (1979). Assay mixtures of $1.25 \mathrm{ml}$ contained: $1 \mathrm{ml} 0.2 \mathrm{M}$-Tris buffer, $\mathrm{pH} 7.2 ; 1 \mathrm{mM}-\mathrm{CaCl}_{2}$; $0.1 \%$ azocasein; and $0.25 \mathrm{ml}$ enzyme sample (diluted when necessary). They were incubated for $1 \mathrm{~h}$ at $35{ }^{\circ} \mathrm{C}$, and the reaction was terminated by the addition of $1 \mathrm{ml}$ of $10 \%$ trichloroacetic acid. The samples (triplicates) were 
centrifuged at $6000 \mathrm{~g}$ for $10 \mathrm{~min}$ and $1 \mathrm{ml}$ supernatant was placed in a second tube containing $0 \cdot 25 \mathrm{ml} 1 \mathrm{M}-\mathrm{NaOH}$. Enzyme activity was determined as the increase of absorbance at $420 \mathrm{~nm}$ as compared with a blank containing water instead of the sample. Serine proteinase was measured as the difference between the activity of an untreated sample and one treated with the inhibitor phenylmethylsulphonyl fluoride $\left(10^{-3} \mathrm{M}, 30\right.$ min preincubation). One unit was defined as a change in absorbance of 1.0 . The concentration of leukaemomycin in the medium was assayed by the agar plate diffusion assay with Bacillus subtilis ATCC 6633 as test organism.

[ $\left.\mathrm{U}^{14} \mathrm{C}\right]$ Acetate and $\mathrm{KH}_{2}{ }^{32} \mathrm{PO}_{4}$ incorporation studies into lipids and lipid turnover. Mycelia were harvested after $24 \mathrm{~h}$ by suction filtration, washed with $0.9 \% \mathrm{NaCl}$ and resuspended in the same amount of fresh Hickey-Tresner's medium supplied with approx. $2 \mathrm{MBq}\left[\mathrm{U}-{ }^{14} \mathrm{C}\right]$ acetate or, alternatively, $0.3 \mathrm{MBq} \mathrm{KH}_{2}{ }^{32} \mathrm{PO}_{4}$. After 5 h on a rotary shaker $\left(29^{\circ} \mathrm{C}\right)$ in $500 \mathrm{ml}$ flasks, the mycelia were harvested by suction filtration, washed with unlabelled $0 \cdot 1 \mathrm{M}$ sodium acetate or $0.1 \mathrm{M}-\mathrm{KH}_{2} \mathrm{PO}_{4}, \mathrm{pH} 7 \cdot 0$, and extracted twice with $100 \mathrm{mlCHCl} / \mathrm{methanol}(2: 1, \mathrm{v} / \mathrm{v})$ in order to isolate the total lipid fraction. It was then either measured by means of a LKB model 81000 LSC counter using toluene/PPO/POPOP as the scintillation cocktail or samples were applied to TLC plates (silica gel) according to mycelial dry weight and developed by the three different solvent systems described above. Autoradiograms were exposed for at least 3 weeks. After that time, the TLC plates were stained with iodine vapour and the layers of silica gels containing the spots of triglycerides and polar lipids were scraped off, transferred to scintillation vials and measured. For the estimation of lipid turnover, mycelium was incubated for $5 \mathrm{~h}$ with [U-14 C]acetate, washed and resuspended in fresh medium without further labelling and shaken for an additional period of $5 \mathrm{~h}$. After that time the lipids were extracted and the radioactivity estimated.

Reproducibility of the results. All results were reproduced in at least three independent series of cultivations. It proved necessary to circumvent the relatively low genetic stability of the indicator mutants 86 and 15 by selection of those clones from surface agars which displayed a sufficiently sensitive response to the addition of inducers I or II in forming aerial mycelia and anthracyclines.

\section{RESULTS}

\section{Effects of the autoregulatory inducer II on vegetative development and differentiation of submerged cultures of blocked mutants of $S$. griseus}

Mutant $86\left(\mathrm{Amy}^{-} \mathrm{Lkm}^{-} \mathrm{Are}^{-}\right)$was selected as a prototrophic derivative from mutagenized spores of the parental strain S. griseus JA $5142\left(\right.$ Amy $^{+} \mathrm{Lkm}^{+}$Are $^{+}$) while mutant 15 was isolated from another, anthracycline-producing strain, JA 3933, being very similar in its properties to JA 5142. In contrast to the ancestral strains, the mutants 86 and 15 were unable to form both aerial mycelia and leukaemomycin throughout surface cultivation (Gräfe et al., 1982a) but in the presence of I, II or similarly structured inducers (Gräfe et al., 1982b; Gräfe et $a l ., 1983 a, b$; Gräfe \& Eritt, 1983) they developed normal signs of cytodifferentiation in surface cultures, e.g. production of both spores and antibiotics. The blocked mutants did not differ from the parental strain JA 5142 (and JA 3933, not shown here) in their growth rate on HickeyTresner's medium, but under these submerged conditions production of anthracycline pigments (leukaemomycin) was not observed (Fig. 1). Formation of the antibiotic and its inactive congener pigments, concomitant with pronounced changes in the mycelial morphology, was induced by addition of II at zero time $\left(0 \cdot 5-10 \mu \mathrm{g} \mathrm{ml}^{-1}\right)$. TLC investigations confirmed that the spectrum of secondary metabolites produced was identical with that of the parental strains JA 5142 and JA 3933.

The parental strains formed extended hyphal aggregates, while blocked mutants developed dense pellets, the margin of which sometimes fragmented, giving rise to the appearance of rodshaped mycelial pieces (Eritt et al., 1983). The presence of the natural autoregulator I or (as shown in this work) the interspecific inducer II from $S$. viridochromogenes (Gräfe et al., 1982b), 'normalized' the shape of the mycelia insofar as long, filamentous hyphae appeared as in the parental strains. These effects coincided with the onset of antibiotic production and became most apparent when II had been administered at zero time of cultivation. The induction of differentiation by II was dependent on its concentration in the range of $0 \cdot 1-2 \mu \mathrm{g} \mathrm{ml}^{-1}$. To get more reproducible results, we used an excessive concentration of II $\left(10 \mu \mathrm{g} \mathrm{ml}^{-1}\right)$ throughout these experiments. A bioassay (Eritt et al., 1982; Gräfe \& Eritt, 1983) has proved that only $10 \%$ of added II is taken up from the mycelia in the course of $96 \mathrm{~h}$ cultivation. Because the natural autoregulator, I, possesses about a tenfold higher specific activity towards mutants 86 and 15 than II (Gräfe et al., 1982b), it appears likely that II will be oxidized to I within the cells prior to 

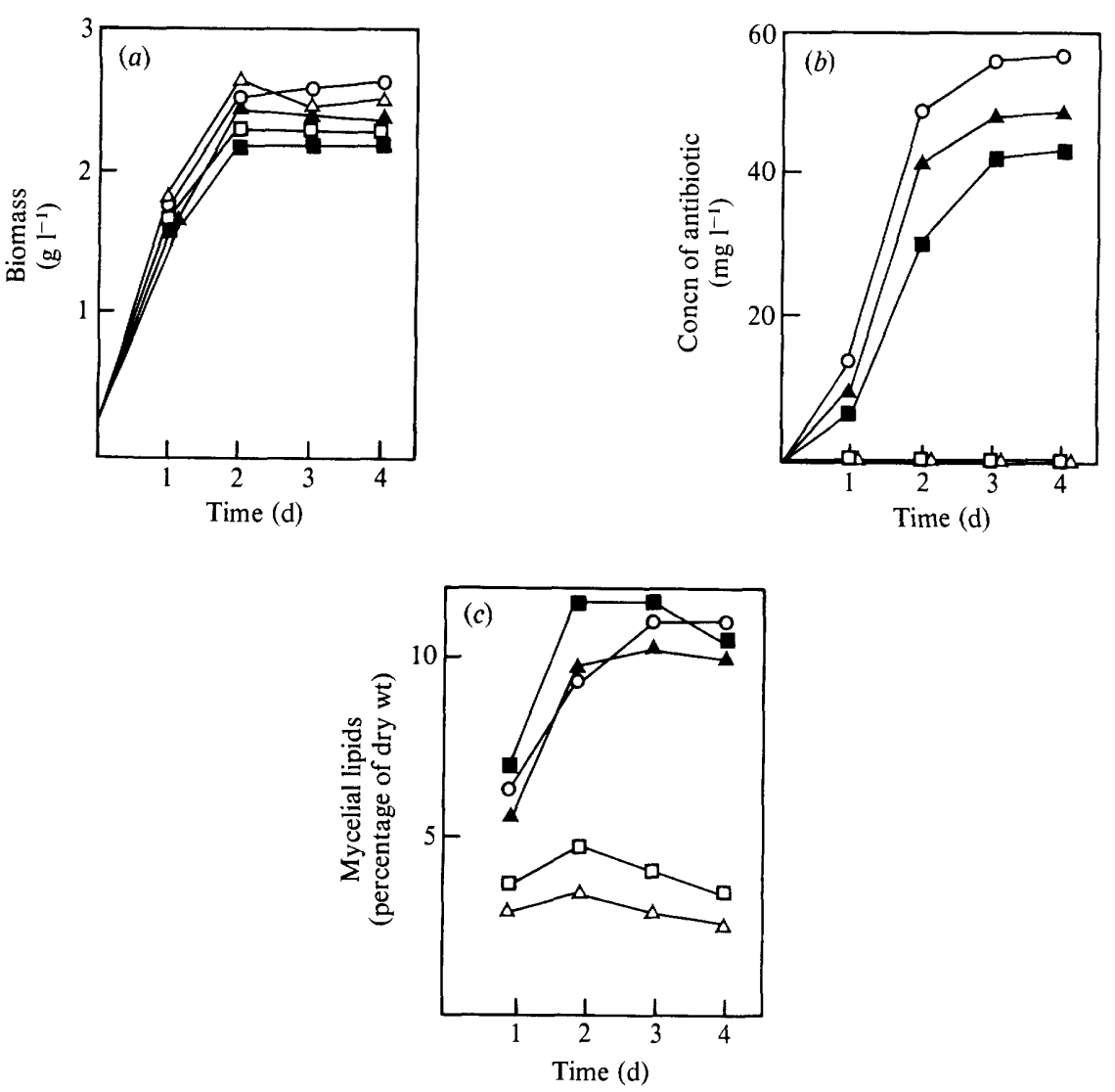

Fig. 1. (a) Growth of strains of S. griseus; (b) production of leukaemomycin and (c) content of mycelial lipids. $\bigcirc$, Parental strain JA 5142; $\square$, blocked mutant $86 ; \triangle$, blocked mutant 15 . Filled symbols, autoregulator II added at zero time; open symbols, II omitted; the mean error in lipid assays was approx. $\pm 1 \%$.

exerting its influence on mycelial structure. Furthermore, addition of $10 \mu \mathrm{g} \mathrm{II} \mathrm{ml}^{-1}$ to both parental strains resulted in no detectable change in their morphology and antibiotic production, suggesting that they can produce abundant amounts of the endogenous effector, I.

\section{Influence of inducer II on the formation and composition of lipids}

In comparison with the ancestral strain JA 5142 (and JA 3933, not shown here), the blocked mutants 86 and 15 displayed greatly reduced levels of lipid material, but addition of II at zero time restored the normal amount of solvent-extractable products (Figs $1 c, 2$ ). By means of ${ }^{14} \mathrm{C}$ and ${ }^{32}$ P-labelling we could show that the higher content of lipids in the parental strain JA 5142 resulted merely from an elevated rate of synthesis of both neutral and polar lipids and not from the reduced rate of their turnover. Thus, after $5 \mathrm{~h}$ incubation of mycelia prelabelled with [U${ }^{14} \mathrm{C}$ lacetate the turnover of lipids was $39 \%$ and $33 \%$ in the parental strain JA 5142 and mutant 86 , respectively. Growth of mutants 86 and 15 for $24 \mathrm{~h}$ in the presence of II greatly increased the rate of lipid synthesis up to the parental level, but its addition at zero time was necessary to show this effect. Labelling experiments showed that incubation of mycelia obtained from $24 \mathrm{~h}$ cultures with II for $5 \mathrm{~h}$ did not stimulate lipid formation, suggesting that it interfered with the formation, rather than with the activity, of the enzymes of lipid and polyketide biosynthesis.

The higher rate of lipid synthesis in 24 h cultures of the parental strain JA 5142 (and JA 3933) and mutant 86 (and 15) following the addition at zero time of autoregulator II can be attributed to the increase in both neutral lipids and polar phospholipids, but the relatively higher 


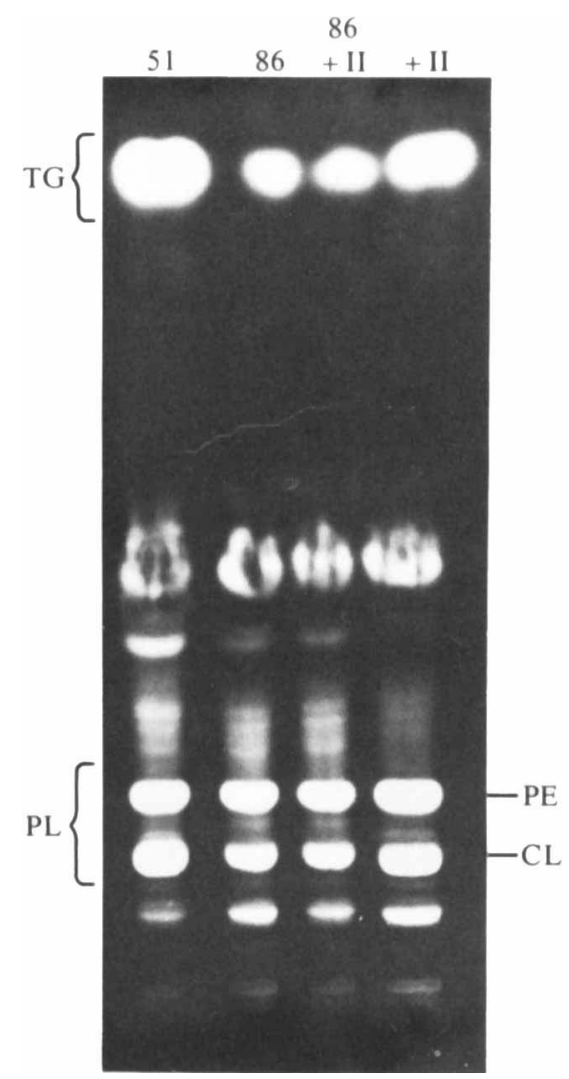

Fig. 2. Autoradiogram of total [U-14 C]acetate-labelled lipids from mycelia after $29 \mathrm{~h}$ growth. Samples were applied according to the mycelial dry weight, and the chromatogram was developed by three subsequent runs in different solvent systems. 51, parental strain JA 5142;86, mutant 86;86 + II, mutant 86 supplied with II throughout the $5 \mathrm{~h}$ incorporation experiment; + II, mutant 86 grown for $24 \mathrm{~h}$ in the presence of II and supplied with II throughout the incorporation experiments. The relative incorporations (c.p.m.) into the zone of triglycerides (TG) and phospholipids (PL) amounted to: 51 , 6158 (TG) and 984 (PL); 86, 1100 (TG) and 2000 (PL); $86+$ II, 1200 (TG) and 2100 (PL); + II, 5163 (TG) and 895 (PL). CL, cardiolipin; PE, phosphatidylethanolamine.

incorporation of $\left[\mathrm{U}-{ }^{14} \mathrm{C}\right]$ acetate into the triglycerides indicated a relative decrease in the proportion of the polar lipids. This conclusion has been further supported by TLC studies showing decreased amounts of phospholipids and ornithinolipids in the mycelial lipid fraction of the parental strain JA 5142 (and JA 3933) and mutants 86 and 15 grown in the presence of II (Fig. 3), in agreement with earlier findings with surface cultures (Gräfe et al., 1983c).

\section{Effect of inducer II on the composition of mycelial fatty acids}

The non-differentiating mutants 86 and 15 were distinguishable from the parental strain JA 5142 by minor changes in the ratios of individual fatty acids, e.g. a higher proportion of 12methyltetradecanoic acid $\left(\mathrm{aC}_{15: 0}\right)$ in relation to isopalmitic acid $\left(\mathrm{iC}_{16: 0}\right)$ (Table 1). In no instance did the addition of II reconstitute the parental spectrum of fatty acids, suggesting that cytodifferentiation of mutants 86 and 15 does not need the proper adaptation of their fatty acids to the parental spectrum and that these autoregulator-negative (Are ${ }^{-}$) mutants harbour additional genetic changes affecting mainly the formation of the methyl-branched fatty acids (Gräfe et al., 1982c). 

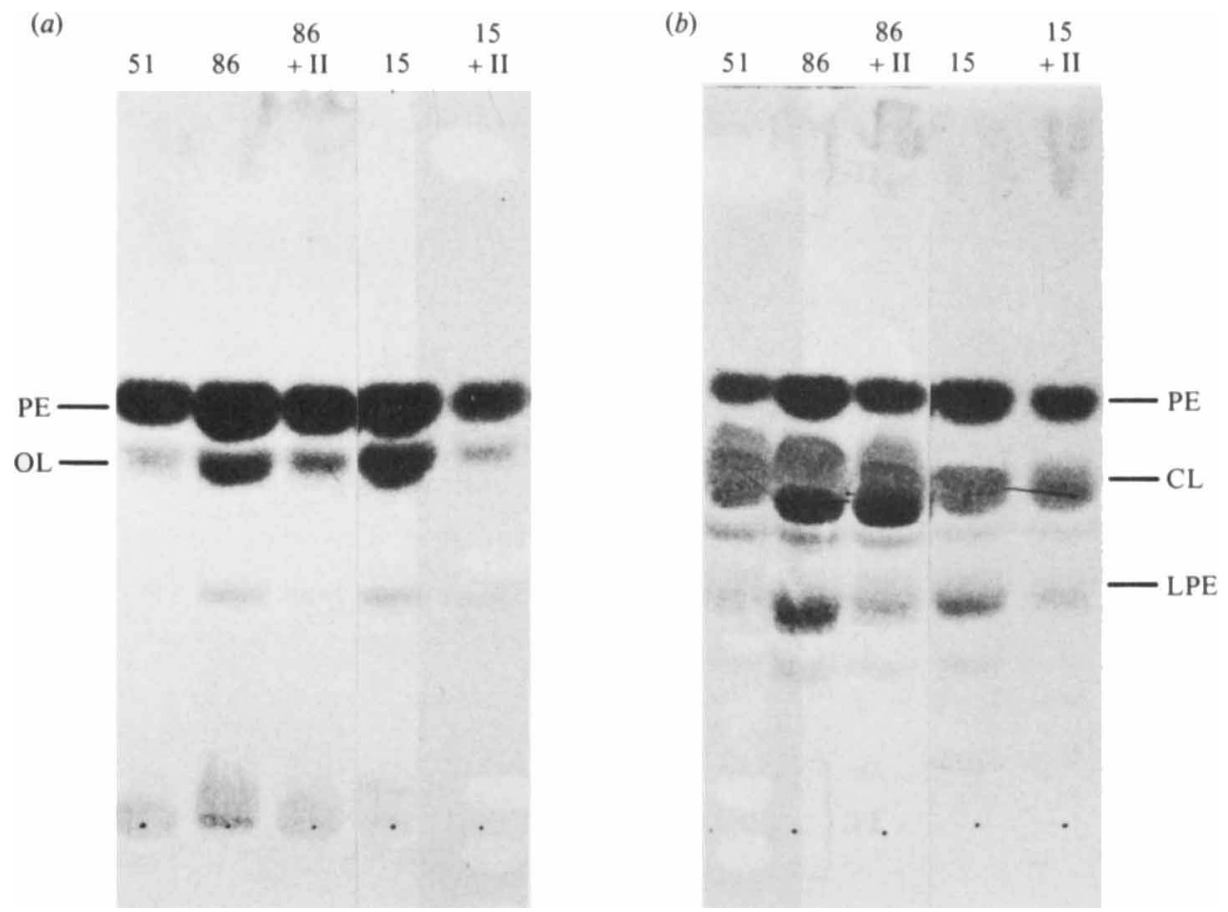

Fig. 3. Comparison of the relative amounts of polar lipids within the total pool of mycelial lipids from cultures after $48 \mathrm{~h}$ growth. (a) Aminolipids; (b) phospholipids: Application: $200 \mu \mathrm{g}$ lipid material per sample. PE, phosphatidylethanolamine; CL, cardiolipin; OL, ornithinolipid; LPE, lysophosphatidylethanolamine. 51, parental strain JA 5142;86 and 15, blocked mutants; $86+$ II and $15+$ II, blocked mutants grown in the presence of autoregulator II.

Table 1. Relative proportions of fatty acids from the mycelia of the parental strain JA 5142 $\left(\mathrm{Amy}^{+} \mathrm{Lkm}^{+} \mathrm{Are}^{-}\right)$and the blocked $\left(\mathrm{Amy}^{-} \mathrm{Lkm}^{-}\right.$Are $\left.{ }^{-}\right)$mutants 86 and 15

Autoregulator II was added at zero time throughout the pertinent experiments.

Fatty acids (percentage of total)*

\begin{tabular}{|c|c|c|c|c|c|c|c|c|c|c|c|c|c|}
\hline \multirow[b]{2}{*}{ Strain } & \multirow[b]{2}{*}{ Time (h) } & \\
\hline & & i1 4:0 & $14: 0$ & i15:0 & a15:0 & $15: 0$ & i16:0 & $16: 0$ & $16: 1$ & a17:0 & UI & $18: 0$ & $18: 1$ \\
\hline \multirow[t]{3}{*}{ JA 5142} & 24 & $4 \cdot 4$ & $1 \cdot 1$ & $7 \cdot 7$ & $30 \cdot 8$ & tr. & $22 \cdot 6$ & $11 \cdot 0$ & $3 \cdot 3$ & $9 \cdot 9$ & $5 \cdot 5$ & $3 \cdot 0$ & $1 \cdot 1$ \\
\hline & 48 & $4 \cdot 0$ & 0.7 & $5 \cdot 4$ & $26 \cdot 0$ & $2 \cdot 7$ & $23 \cdot 5$ & $11 \cdot 0$ & $3 \cdot 7$ & $10 \cdot 0$ & $9 \cdot 0$ & 1.7 & $2 \cdot 3$ \\
\hline & 72 & $4 \cdot 2$ & 0.7 & $3 \cdot 2$ & $29 \cdot 2$ & 3.9 & $25 \cdot 0$ & 9.8 & $2 \cdot 6$ & $11 \cdot 7$ & $7 \cdot 0$ & $2 \cdot 0$ & $1 \cdot 3$ \\
\hline \multirow[t]{3}{*}{86} & 24 & $3 \cdot 2$ & $2 \cdot 8$ & 0.6 & $27 \cdot 0$ & 4.8 & $12 \cdot 1$ & $15 \cdot 8$ & $2 \cdot 8$ & $7 \cdot 4$ & $9 \cdot 1$ & $6 \cdot 1$ & $7 \cdot 8$ \\
\hline & 48 & $4 \cdot 7$ & 0.6 & $4 \cdot 7$ & $34 \cdot 2$ & $4 \cdot 7$ & $17 \cdot 1$ & $7 \cdot 1$ & $4 \cdot 1$ & $10 \cdot 6$ & $8 \cdot 3$ & $1 \cdot 2$ & $2 \cdot 3$ \\
\hline & 72 & $4 \cdot 3$ & 1.6 & $3 \cdot 1$ & $33 \cdot 5$ & $7 \cdot 0$ & 14.8 & $9 \cdot 4$ & 1.6 & $9 \cdot 4$ & $6 \cdot 6$ & $4 \cdot 7$ & 3.9 \\
\hline \multirow[t]{3}{*}{$86+$ II } & 24 & 6.7 & tr. & tr. & $43 \cdot 3$ & $6 \cdot 3$ & $19 \cdot 8$ & $4 \cdot 7$ & $3 \cdot 3$ & 9.9 & $5 \cdot 5$ & tr. & tr. \\
\hline & 48 & 1.4 & 0.9 & tr. & $34 \cdot 2$ & $4 \cdot 5$ & $12 \cdot 6$ & $8 \cdot 1$ & $4 \cdot 5$ & $13 \cdot 5$ & $12 \cdot 0$ & $3 \cdot 0$ & $4 \cdot 6$ \\
\hline & 72 & $2 \cdot 7$ & $1 \cdot 1$ & tr. & 36.4 & $4 \cdot 6$ & 15.5 & 6.8 & 1.8 & $13 \cdot 6$ & 11.0 & 1.8 & $4 \cdot 6$ \\
\hline & 48 & $4 \cdot 4$ & $1 \cdot 4$ & $5 \cdot 8$ & $32 \cdot 0$ & 4.9 & $17 \cdot 0$ & $7 \cdot 8$ & $4 \cdot 8$ & $8 \cdot 7$ & 9.7 & $2 \cdot 9$ & tr. \\
\hline $15+$ II & 48 & $4 \cdot 5$ & 1.6 & $5 \cdot 3$ & 30.0 & 3.6 & $20 \cdot 3$ & $7 \cdot 3$ & $4 \cdot 1$ & $11 \cdot 3$ & $10 \cdot 5$ & 1.6 & tr. \\
\hline
\end{tabular}

* Abbreviations: i14:0, 12-methyltridecanoic acid; 14:0, tetradecanoic acid; i15:0, 13-methyltetradecanoic acid; a 15:0, 12-methyltetradecanoic acid; $15: 0$, pentadecanoic acid; i16:0, isopalmitic acid; $16: 0$, palmitic acid; $16: 1$, hexadecenoic acid; a17:0, 14-methylhexadecanoic acid; UI, unidentified; 18:0, stearic acid; $18: 1$, oleic acid; tr., traces.

Deviations from the values given may be a maximum of $\pm 5 \%$. 

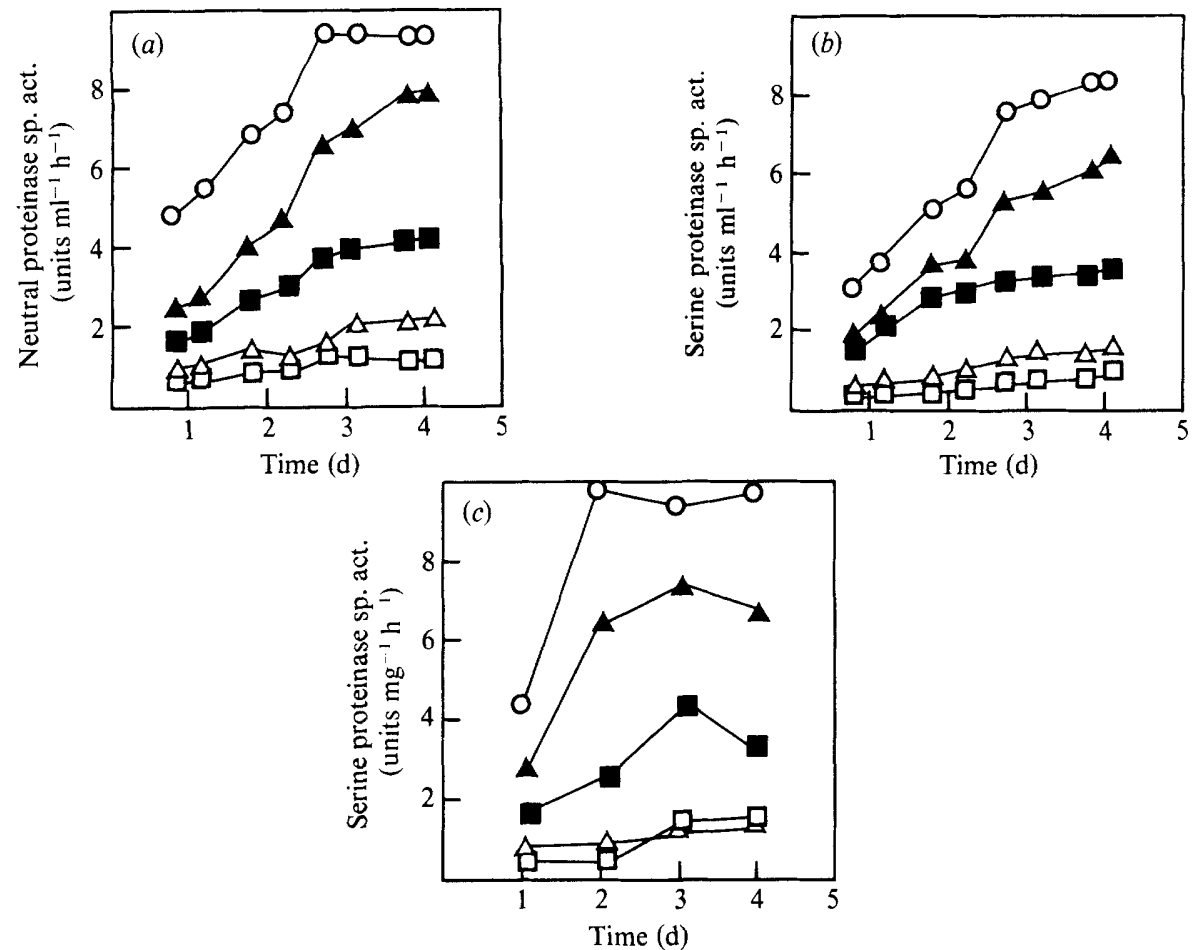

Fig. 4. Extracellular and mycelial levels of neutral proteinases. (a) Neutral proteinase in the culture medium; (b) serine proteinase in the culture medium; (c) serine proteinase in the mycelium. $\bigcirc$, Parental strain JA $5142 ; \square$, mutant $86 ; \triangle$, mutant 15 . Filled symbols : autoregulator II added at zero time; open symbols, no II present.

\section{Effect of inducer II on cytochromes from mutants 86 and 15}

In contrast to our recent findings with surface cultures, which indicated a profound effect of autoregulators such as I and II on the cytochrome spectrum of mutant 86 (Gräfe et al., 1983 b), the addition of II to submerged cultures of mutants 86 and 15 did not cause any alteration in the ratio of $b$ - to $c$-type cytochromes. Thus, an almost identical maximum wavelength of the Soret absorption at 431 and $430 \mathrm{~nm}$, respectively, was found with the mycelia grown either in the presence or in the absence of II. This result is compatible with the view that differences in the composition of the respiratory chain exist only between substrate and aerial mycelia.

\section{Effect of inducer II on production of $N A D(P)$-glycohydrolase and neutral proteinases}

As previously shown (Gräfe et al., 1981), addition of II to surface cultures of mutant 86 and the concomitant restoration of cytodifferentiation was not accompanied by increased production of NAD(P)-glycohydrolase up to the large amounts present in the medium and the mycelium of the parental strain JA 5142. These investigations were done because partial reconstitution of enzyme production by A-factor (I) has been reported for the blocked mutants of streptomycinproducing strains of $S$. griseus (Voronina et al., 1978; Hara \& Beppu, 1982a,b), and there appeared to be a correlation between the activity of this enzyme and the formation of macrotetrolides (Bröker \& Pape, 1981). In the case of the submerged cultures of mutants 86 and 15 we confirmed our earlier results, showing that addition of II (or I) did not increase enzyme synthesis in the mycelium or its secretion into the medium, though high activities ( $>40 \mathrm{nkat} \mathrm{ml}^{-1}$ ) were present in the medium of the parental strain JA 5142 and JA 3933 and also in their mycelia.

Apart from the differences in $\mathrm{NAD}(\mathrm{P})$-glycohydrolase between the ancestral strains and blocked mutants 86 and 15 , there were also apparent differences in the capacity to form neutral proteinases (Fig. 4). The presence of II stimulated both their formation in the mycelium of the blocked mutants and their secretion into the medium. 


\section{DISCUSSION}

The beneficial effect of butyrolactone-type autoregulators from streptomycetes, for example trans-2-(6'-methylheptanol-1'-yl)-3-hydroxymethyl-4-butanolide (II) on differentiation of nonsporulating, antibiotic-negative mutants 86 and 15 of anthracycline-producing, differentiating strains of $S$. griseus established that a phenotype altered by mutation can be almost completely repaired when the product of the deleted gene function has been added to the medium. It can reasonably be assumed that the incapacity of mutants 86 and 15 to differentiate results chiefly from a single mutation affecting the synthesis of the natural autoregulator (I) and not from any other mutations concerned with the regulation of fatty acid composition. Mutations leading to deletion of autoregulator synthesis may occur frequently, since the same type of blocked, autoregulator-negative mutant was obtained from two different strains of anthracyclineproducing $S$. griseus.

The results obtained in the present study suggest that, at least in anthracycline-producing species of $S$. griseus, changes in the rate of lipid synthesis and in the concomitant formation of intracellular and extracellular proteinases are a feature of cytodifferentiation. Possibly the induction of these alterations in the presence of II, or the natural autoregulator, I, triggers further developmental events from the stage of the rapidly growing vegetative mycelium towards differentiated cell types generating polyketide antibiotics such as leukaemomycin. Thus, alterations in the mycelial level of lipids and in their composition will certainly affect many functions of the cytoplasmic membrane, including the secretion of extracellular proteinases. The neutral proteinases in particular have been proposed to play a role in the regulation of spore formation in Bacillus species and streptomycetes (Murao et al., 1982; Ginther, 1979; Aharonowitz, 1980).

Identification of the cellular target structure for the autoregulatory butyrolactones clearly awaits further elucidation, but from the results of this communication it appears likely that I, II and similar inducers govern cellular development as pleiotropic effectors at a very early stage. Perhaps they make the cells competent to adapt to particular environmental changes with the onset of specialized cellular responses, leading to the formation of morphologically differentiated mycelia, spores and antibiotics.

We are greatly indebted to Dr A. Steudel (Institute of Technical Chemistry, Leipzig) for recording the cytochrome spectra, and to Mrs C. Karkowski and Mrs M. Pascholdt for their technical assistance.

\section{REFERENCES}

AHARONOWITZ, Y. (1980). Nitrogen-metabolite regulation of antibiotic biosynthesis. Annual Review of Microbiology 34, 209-233.

Biro, S., Vitalis, S. \& Szabo, G. (1980). A substance effecting differentiation in Streptomyces griseus. Purification and properties. European Journal of Biochemistry 103, 359-363.

BRÖKER, M. \& PAPE, H. (1981). NADP-glycohydrolase and macrotetrolide production in Streptomyces griseus. In Actinomycetes, pp. 447-459. Edited by K. P. Schaal \& G. Pulverer. Stuttgart: G. Fischer.

Chater, K. F. \& Merrick, M. J. (1976). Approaches to the study of differentiation in Streptomyces coelicolor. In Genetics of Industrial Microorganisms, pp. 583-589. Edited by K. D. MacDonald. London : Academic Press.

ERITT, I., GräFe, U. \& FleCK, W. F. (1982). A screening method for autoregulators of anthracycline-producing streptomycetes. Zeitschrift für allgemeine Mikrobiologie, 22, 91-95.

Eritt, I., Elske, G., Gräfe, U. \& Fleck, W. F. (1983). Influence of low molecular weight stimulators on differentiation of Streptomyces griseus. In
Proceedings of the International Symposium on Genetics and Differentiation of Streptomycetes (held in Weimar, GDR, May 2-5, 1983), pp. 61. Jena, GDR: Central Institute of Microbiology.

ERITt, I., Reinhardt, G., GräFe, U. \& Fleck, W. F. (1984). Inducers of both cytodifferentiation and anthracycline biosynthesis of Streptomyces griseus and their occurrence in actinomycetes and other microorganisms. Zeitschrift für allgemeine Mikrobiologie 24 (in the Press).

FleCK, W. F. \& STRAuSs, D. (1975). Leukaemomycin, an antibiotic with antitumor activity. 1. Screening, fermentation and biological activity. Zeitschrift für allgemeine Mikrobiologie, 15, 495-503.

GINTHER, C. L. (1979). Sporulation and the production of serine proteinase and cephamycin C by Streptomyces lactamdurans. Antimicrobial Agents \& Chemotherapy 15, 522-526.

GRÄFE, U. \& ERITT, I. (1983). On the biological inactivity of 4,5-dihydroxy-n-decanoic acid-4-lactones. Journal of Antibiotics 36, 1592-1593.

GräFE, U., ERITT, I. \& FlECK, W. F. (1981). Evidence against a general role of NADP-glycohydrolase in 
cytodifferentiation of Streptomyces griseus. Journal of Antibiotics 34, 1385-1387.

Gräfe, U., Rfinhardt, G., Schade, W., Krebs, D., ERITt, I., Fleck, W. F., HeinRICH, E. \& Radics, L. $(1982 a)$. Isolation and structure of novel autoregulators from Streptomyces griseus. Journal of Antibiotics 35, 609-614.

Gräfe, U., Schade, W., Eritt, I., Fleck, W. F. \& RADICS, L. (1982b). A new inducer of anthracycline biosynthesis from Streptomyces viridochromogenes. Journal of Antibiotics 35, 1722-1723.

Gräfe, U., Reinhardt, G., KREBS, D., Roth, M. \& NoACK, D. (1982c). Altered lipid composition in a non-differentiating derivative of Streptomyces hygroscopicus. Journal of General Microbiology 128, 26932698.

Gräfe, U., Reinhardt, G., ERItT, I., Schade, W., FleCK, W. F. \& Radics, L. (1983a). Interspecific inducers of cytodifferentiation and anthracycline biosynthesis from Streptomyces bikiniensis and cyaneofuscatus. Biotechnology Letters 5, 591-596.

GRÄFE, U., ReINHARDT, G., ERITT, I., FleCK, W. F. \& STEUDEL, A. $(1983 b)$. Effect of the autoregulator from Streptomyces griseus on surface cultures of the blocked mutant JA 5142/86. Zeitschrift für allgemeine Mikrobiologie 23, 559-565.

HARA, O. \& BEPPU, T. (1982a). Mutants blocked in streptomycin production in Streptomyces griseus the role of A-factor. Journal of Antibiotics 35, 349358.

HARA, O. \& BEPPU, T. (1982b). Induction of streptomycin-inactivating enzyme by A-factor in Streptomyces griseus. Journal of Antibiotics 35, 1208-1218.
HopwOOD, D. A. (1983). Actinomycete genetics and antibiotic production. In Biochemistry and Genetic Regulation of Commercially Important Antibiotics, pp. 1-23. Edited by L. C. Vining. Reading, Mass.: Addison-Wesley Publishing.

Kalakoutski, L. V. \& Agre, N. S. (1976). Comparative aspects of development and differentiation in actinomycetes. Bacteriological Reviews 40, 469524.

KHOKHLOV, A. S. (1982). Low molecular weight microbial bioregulators of secondary metabolism. In Overproduction of Microbial Products (FEMS Symposium no. 13), pp. 97-109. Edited by V. Krumphanzl, B. Sikyta \& Z. Vanek. London: Academic Press.

Kleiner, E. M., Onoprienko, V. V., Pliner, S. A., SOIFER, V. S. \& KHoKhlov, A. S. (1977). The synthesis of racemic A-factor, a bioregulator from Streptomyces griseus. Bioorganic Chemistry 3, 424426.

Murao, S., Shimizu, Y., Kameda, M. \& Nishino, T. (1982). Inhibition of sporulation of genus Bacillus by various microbial protease inhibitors. Agricultural and Biological Chemistry 46, 3075-3077.

POGELL, B. M. (1979). Regulation of aerial mycelium formation in streptomycetes. In Genetics of Industrial Microorganisms, pp. 218-224. Edited by O. K. Sebek \& A. L. Laskin. Madison, Wisconsin: American Society for Microbiology.

Voronina, O. I., Tovarova, I. I. \& Khokhlov, A. S. (1978). Studies on the A-factor induced inhibition of glucose-6-phosphate dehydrogenase in Actinomyces streptomycini. Bioorganic Chemistry 4, 15381546. 